\title{
Small Heterocyclic Molecules as Anticancer Agents: Design, Syntheses and Evaluation Against MCF-7 Cell Lines
}

\section{Roshni Varshney}

Babu Banarasi Das Northern India Institute of Technology

\section{Vimlesh Kumar}

Babu Banarasi Das Northern India Institute of Technology

\section{Gul Naz Fatima}

Babu Banarasi Das Northern India Institute of Technology

Shailendra K. Saraf ( $\sim$ dirpharmniec@gmail.com )

Babu Banarasi Das Northern India Institute of Technology https://orcid.org/0000-0002-0569-1213

\section{Research Article}

Keywords: Isatin, substituted isatin, Mannich base, breast cancer, MCF-7 cell line, percentage cell viability, in-vitro, molecular docking, in-silico ADMET prediction.

Posted Date: February 2nd, 2022

DOI: https://doi.org/10.21203/rs.3.rs-1295387/v1

License: (ㄷ) (i) This work is licensed under a Creative Commons Attribution 4.0 International License. Read Full License 


\section{Abstract}

Isatin and substituted isatin derivatives possess a broad spectrum of biological activities. These moieties also have a wide scope for substitution, particularly at positions N-1 and C-3. Therefore, the study focused at synthesizing some novel isatin/substituted isatin Schiff's bases and screening them for anti-cancer activity against MCF-7 cell lines. All the compounds were synthesized by conventional methods in a two-step synthesis. Mannich bases of isatin were synthesized by reacting secondary amines and $37 \%$ formalin, which were then reacted with primary aromatic amines in the presence of glacial acetic acid to yield the title compounds. All the compounds were characterized by physical, chromatographic and spectroscopic methods. The synthesized analogues showed good activity against MCF-7 cell lines when compared to the standard drug, Doxorubicin. At a concentration of $10 \mu \mathrm{g} / \mathrm{ml}$, the analogues IDF 3B, IDF 3G, IDF 3 H and IDF 3 I showed percentage cell viability of $35.46227 \pm 12.92,34.37412 \pm 5.39,35.81035 \pm 5.25,36.19748 \pm 3.27$ respectively compared to percentage cell viability of $22.36828 \pm 2.35$ for the standard. The obtained data indicated that the analogues were effective against breast cancer, and therefore the isatin moiety could serve as the lead to the development of new anti-breast cancer agents.

\section{Introduction}

Cancer is a collection/group of cells characterized by their uncontrolled growth and division. It is the second leading cause of death worldwide and exerts tremendous burden on the health care system [1, 2]. In Asian countries, more than $60 \%$ breast cancer cases are diagnosed as estrogen receptor a positive (ERa) cancers. In normal mammary gland development, ERa plays a significant role in breast cancer development [3]. Among all types of cancer, breast cancer is one of the most common cancers occurring especially in the females.

Isatin ( $1 \mathrm{H}$-Indole-2,3-dione) is an indole oxidized derivative belonging to the class of heterocyclic molecules [4]. It possesses several biological activities viz. anti-fungal [5], anti-tubercular [6], anti-HIV, anti-viral [5], anti-cancer [7-9], anticonvulsant [10], anti-bacterial [11], anti-inflammatory [12], and anti-oxidant [13]. A number of isatin analogues may be obtained by substitutions at different positions to potentiate the cytotoxic and anticancer effectiveness [14].

Also, benzothiazole possesses a wide variety of biological activities [15-22]. 2-aminobenzothiazole has many applications in medicinal and bioorganic chemistry [23]. In addition, pyrimidine is a well-known bio-active scaffold [2431]. Both the moieties are found to exhibit cytotoxic effect against various human cancer cell lines [32, 33] These nucleuses in combination with particularly with isatin have been found active against breast cancer [34-36]. Considering the above-mentioned facts, it was decided to synthesize conjugates of isatin and benzothiazole/pyrimidine moieties, in anticipation that the new compounds may have synergistic effect against the breast cancer cell-lines.

\section{Results And Discussion}

A novel series of conjugates of isatin/substituted isatin derivatives and heterocyclic moieties were designed using molecular docking simulations. The most molecules displaying good binding affinity were selected. These were synthesized in wet-laboratory through a two-step reaction scheme as given in Scheme 1. The compounds obtained were purified by column chromatography and their physico-chemical parameters were studied. The structures of the synthesized derivatives were confirmed by spectral techniques [37] viz. FTIR spectroscopy, mass spectrometry, ${ }^{1} \mathrm{H}-\mathrm{NMR}$, ${ }^{13} \mathrm{C}-\mathrm{NMR}$ spectroscopy, and elemental analysis.

Through endocrine study hyper-production of estrogen hormone has been of the leading causes for development of the breast cancer. Human being naturally contains two estrogen receptors, namely ER- $a$ and ER- $\beta$. These predominantly are expressed in the mammary gland and uterus; and play critical role in metabolism, proliferation and maturation of the 
breast cancer cells. The receptor, ER- $a$ is well established to be involved in monitoring of the immune response, retarded apoptosis, in metastasis and in cell growth [38]. Hyper activity of estrogen may potentially lead to rise in the number of ER-a receptor in the mammalian cells which may lead to development of various types of breast cancers [39, 3].

Since, this may be considered as one of the important molecular targets of development of anti-breast cancer drugs, therefore, it was decided to take this protein (Human estrogen receptor alpha, PBD ID 3ERT) and explore the molecular interactions with the synthesized ligand molecules. Another target protein, (Human progesterone receptor, PDB ID 4OAR) was also selected to dock the synthesized ligands and determine their binding scores. The hormone plays a key role in reproductive function in women. It is found to have diverse application in the female health and in treatment of hormone-related conditions including breast-cancer. The binding energy values for the synthesized compounds IDF $\mathbf{3 ( A -}$ L) were found to lie majorly in the range of -12.13 to $-8.05 \mathrm{kcal} / \mathrm{mol}$. The binding energies for the fifty best conformer poses of compounds were evaluated, and the best pose of compounds was energetically filtered. The compound IDF $\mathbf{3 G}$ displayed the lowest binding energy. Hydrogen-bonding interactions between the pyrimidine ring and the amino acid residue Glu 695 and Ser 728, a pi-pi T-shaped interaction between the isatin ring and Val 726, Phe 778, and a pi-alkyl interaction between diphenyl ring system and Met 759, Leu 718, and a pi-sulfur interaction between benzothiazole and Arg 766 were the important bindings observed for the compounds against the target protein 40AR. While for the protein 3ERT, a hydrogen-bond between hydrogen atom of pyrimidine ring and Asp 351 and Glu 380 was observed. Other important non-covalent interactions observed were pi-anion, pi-donor, pi-sigma, and pi-cation with Trp 383, Lue 525, Ala 350 and Lys 891. (Figure 2) The compounds IDF 3C, IDF 3H and IDF 3I displayed higher binding energies against the both target proteins, thereby suggesting their weaker interactions with the receptor site. Thus, the in-silico molecular docking studies helped to predict binding affinities of the compounds for the target proteins, and hence, the compounds were further studied for their in-vitro potentials.

The synthesized compounds were tested in-vitro for their anti-breast cancer activity against MCF-7 cell line by MTT assay (Table 1). All the tested compounds showed good to moderate activity against the cell line. The compounds IDF 3B, IDF 3C, IDF 3G, IDF 3H and IDF 3I were significantly more effective in decreasing cell viability of MCF-7 cell lines at all concentrations compared to rest of the tested compounds. (Figure 3) From the results obtained, it was found that the compound's ability to decrease the percentage of viable cells was in a concentration gradient manner.

\section{Structure-activity relationship}

The study also helped to established the structure-activity relationship in the series. It was concluded that the compounds bearing the bromoisatin as the primary nucleus, namely IDF $\mathbf{3 G}$, IDF $\mathbf{3} \mathbf{H}$ and IDF $\mathbf{3}$, were found to be more potent than the compounds containing the isatin nucleus, IDF 3(A-D). The results also helped to conclude that the compounds (IDF 3B, IDF 3H) bearing piperazine at position $\mathrm{N}-1$ and pyrimidine moiety at position $\mathrm{C}-3$ of isatin/bromoisatin were more effective, with percentage cell viability of $35 \%$ each at a concentration of $10 \mu \mathrm{g} / \mathrm{mL}$, than the compounds bearing diphenylamine at position $\mathrm{N}-1$ and pyrimidine moiety at position C-3 of the nucleus (IDF 3D, IDF 3J), with percentage cell viability of $48 \%$ and $50 \%$, respectively at concentration of $10 \mu \mathrm{g} / \mathrm{mL}$. 
Table 1

Percentage cell viability of MCF-7 on treatment with the synthesized compounds, IDF 3(A-L).

\begin{tabular}{|llllll|}
\hline Compounds & \multicolumn{2}{l}{ Concentration $(\boldsymbol{\mu g} / \mathrm{ml})$} & 2 & 5 & 10 \\
\cline { 2 - 6 } & Control & $\mathbf{1}$ & & & \\
\hline Doxorubicin & $99.4189 \pm 3.17071$ & $66.3348 \pm 9.1622$ & $53.9813 \pm 4.0154$ & $30.8801 \pm 2.5096$ & $22.3683 \pm 2.3539$ \\
\hline IDF 3A & $100.6812 \pm 1.4661$ & $77.6218 \pm 1.6895$ & $71.3351 \pm 5.4433$ & $60.2499 \pm 8.8545$ & $48.5854 \pm 7.7658$ \\
\hline IDF 3B & $101.0238 \pm 4.8264$ & $75.0815 \pm 10.4512$ & $72.6951 \pm 11.5036$ & $63.8680 \pm 2.8346$ & $35.4623 \pm 12.9189$ \\
\hline IDF 3C & $99.4329 \pm 1.8507$ & $75.1841 \pm 5.9514$ & $71.5147 \pm 10.1972$ & $62.3593 \pm 8.1658$ & $41.8698 \pm 8.0962$ \\
\hline IDF 3D & $96.5333 \pm 1.4661$ & $78.7509 \pm 10.1766$ & $74.9019 \pm 4.1369$ & $68.8973 \pm 4.0644$ & $48.8568 \pm 6.3225$ \\
\hline IDF 3E & $100.4337 \pm 1.5241$ & $74.2604 \pm 3.5563$ & $71.5917 \pm 6.6128$ & $59.0770 \pm 5.3064$ & $48.9595 \pm 3.5967$ \\
\hline IDF 3F & $101.8963 \pm 3.5926$ & $93.8134 \pm 5.3707$ & $89.0662 \pm 3.3749$ & $80.0904 \pm 4.6532$ & $68.6921 \pm 5.2619$ \\
\hline IDF 3G & $100.6848 \pm 1.4661$ & $71.1075 \pm 10.1766$ & $68.9446 \pm 4.1369$ & $48.2261 \pm 4.0644$ & $34.3741 \pm 5.3951$ \\
\hline IDF 3H & $101.0238 \pm 4.8264$ & $65.1361 \pm 3.1249$ & $58.1309 \pm 6.3225$ & $44.1265 \pm 4.5806$ & $35.8104 \pm 5.2540$ \\
\hline IDF 3I & $99.4329 \pm 5.8507$ & $69.4214 \pm 7.6207$ & $61.0004 \pm 3.7419$ & $51.0487 \pm$ & $36.1975 \pm 3.2701$ \\
\hline IDF 3J & $99.5333 \pm 4.0644$ & $77.2626 \pm 6.2929$ & $70.6612 \pm 9.5186$ & $57.3503 \pm 3.9301$ & $50.2960 \pm 8.8654$ \\
\hline IDF 3K & $100.4337 \pm 1.5241$ & $94.4835 \pm 6.2338$ & $90.4277 \pm 3.7419$ & $81.9071 \pm 5.6693$ & $74.7992 \pm 4.1006$ \\
\hline IDF 3L & $101.8963 \pm 3.5926$ & $93.8134 \pm 5.0236$ & $89.2459 \pm 9.5559$ & $89.5538 \pm 6.6772$ & $73.3109 \pm 2.5039$ \\
\hline
\end{tabular}

$\%$ Cell Viability Mean $\pm \mathrm{Sd}$

However, compounds with diphenylamine at N-1 but benzothiazole substitution at C-3 (IDF 3C, IDF 3I) were found to be more potent (\% cell viability $41 \%$ and $36 \%$, respectively). With exception, it was found that compound (IDF 3 G) bearing piperazine at position $\mathrm{N}-1$ and benzothiazole at position $\mathrm{C}-3$ of bromoisatin exhibited highest effective with percentage cell viability of $34 \%$ at $10 \mu \mathrm{g} / \mathrm{mL}$.

Howsoever, the compounds (IDF 3E, IDF 3F, IDF 3K, IDF 3L) that bore morpholine moiety at position N-1 of either isatin or bromoisatin irrespective of the type of substitution at position $\mathrm{C}-3$, were potentially ineffective in decreasing cell-viability of the MCF-7 cells.

The molecular properties of all the synthesized compounds were prevised using Swiss-ADME [40] and admetSAR [41, 42] web servers (Table 1). The OSIRIS Property Explorer and LAZAR applications were used to predict any toxic potential associated with the compounds. The title compounds were found to present no risks of reproductive toxicity, carcinogenicity, irritancy and acute toxicity. (Table 2)

\section{Conclusion}

In the present study, design, syntheses and in-vitro anti-breast cancer activity of novel conjugates of isatin/substituted isatin with heterocyclic moieties have been carried out. All the analogues showed good to moderate activity against the tested MCF-7 anti-cancer cell line. The compounds, IDF 3B, IDF 3 G and IDF 3H were found to be the most potent members in the series. The results obtained from the molecular docking study were also in sync with the in-vitro laboratory result reports. Also, none of the compounds were found to be toxic from the in-silico prediction. The study therefore concludes isatin moiety to be effective and safe, and hence can be used to design more new compounds as effective anticancer agents. 


\section{Materials And Method}

Molecular Docking Studies

AutoDock with its GUI (graphical user interface), AutoDockTools was used to perform the docking simulations [43]. Crystal structures of the target protein was downloaded from the Research Collaboratory for Structural Bioinformatics Protein Data Bank (RCSB PDB). Open Babel GUI was used to convert ChemDraw structure of ligands to their 3D coordinates in the pdb format. Re-dock with the cocrystal ligand was performed to validate the docking calculations. Protein structure was prepared for docking by removal of water molecules and the co-crystallized ligand, followed by addition of polar hydrogen atoms and the partial charges (Kollman and Gasteiger charges), using AutoDockTools 1.5.6. Similarly, ligand structures were prepared, and the files saved in the pdbqt format. For molecular docking, AutoDock 4.2.6 was used. A maximum of fifty conformations were set for each compound using GA (Genetic Algorithm) Lamarckian. 3D analysis of the obtained results was performed for interactions. Discovery Studio Client 2020 was used for the visualization (2D and $3 D$ ) of docked poses of the compounds within the protein target.

General information

The chemicals and reagents were procured from S.D. Fine Chemicals, Rankem, TCl, and Kemphasol. The pre-coated TLC sheets were purchased from Merck, Germany. The solvents were of reagent-grade which were purified and dried by standard procedures.

Table 2: Docking scores and In-silico ADME prediction data of the synthesized compounds, IDF 3(A-L) 


\begin{tabular}{|c|c|c|c|c|c|c|c|c|c|c|c|c|c|}
\hline \multirow{2}{*}{ Comp. } & \multicolumn{2}{|c|}{ Docking scores } & \multirow{2}{*}{$\begin{array}{c}\text { Molecular } \\
\text { weight }\end{array}$} & \multirow{2}{*}{$\begin{array}{c}\text { TPSA } \\
\left(\mathbf{A}^{2}\right)\end{array}$} & \multirow{2}{*}{$\begin{array}{c}\text { Rotatable } \\
\text { bonds }\end{array}$} & \multirow{2}{*}{$\mathrm{HBA}$} & \multirow{2}{*}{ HBD } & \multirow{2}{*}{$\log S$} & \multirow{2}{*}{$\begin{array}{c}\text { GI } \\
\text { absorption }\end{array}$} & \multirow{2}{*}{$\begin{array}{c}\text { P-gp } \\
\text { substrate }\end{array}$} & \multirow{2}{*}{$\begin{array}{l}\text { CYP3A4 } \\
\text { substrate }\end{array}$} & \multirow{2}{*}{$\begin{array}{l}\text { Synthetic } \\
\text { accessibility }\end{array}$} & \multirow{2}{*}{$\begin{array}{c}\begin{array}{c}\text { Violation } \\
\text { of Rule of } \\
\text { Five }\end{array} \\
\end{array}$} \\
\hline & 3ERT & 4OAR & & & & & & & & & & & \\
\hline IDF 3A & -9.28 & -9.55 & 363.44 & 89.07 & 2 & 5 & 1 & -4.64 & High & No & Yes & 3.54 & No \\
\hline IDF 3B & -9.16 & -6.38 & 308.34 & 73.72 & 2 & 6 & 1 & -2.06 & High & Yes & No & 3.23 & No \\
\hline & -11.55 & -12.13 & 460.55 & 77.04 & 5 & 3 & 0 & -8.43 & High & No & Yes & 3.8 & No \\
\hline & -9.58 & -9.41 & 405.45 & 61.69 & 5 & 4 & $b$ & -5.85 & High & No & Yes & 3.44 & No \\
\hline & -9.46 & -9.58 & 378.45 & 86.27 & 3 & 5 & 0 & -4.78 & High & No & Yes & 3.39 & No \\
\hline & -8.91 & -7.01 & 323.35 & 70.92 & 3 & 6 & 0 & -2.19 & High & Yes & No & 3.03 & No \\
\hline & -10.42 & -8.23 & 456.36 & 89.07 & 3 & 5 & 1 & -5.26 & High & Yes & Yes & 3.5 & No \\
\hline & -9.97 & -7.52 & 401.26 & 73.72 & 3 & 6 & 1 & -2.68 & High & Yes & Yes & 3.19 & No \\
\hline & -10.75 & -11.59 & 539.45 & 77.04 & 5 & 3 & 0 & -9.15 & Low & No & No & 3.85 & No \\
\hline & -9.91 & -8.77 & 484.35 & 61.69 & 5 & 4 & 0 & -6.57 & High & No & Yes & 3.55 & No \\
\hline & -9.39 & -9.04 & 457.34 & 86.27 & 3 & 5 & 0 & -5.49 & High & No & Yes & 3.44 & No \\
\hline & -9.53 & -8.05 & 402.25 & 70.92 & 3 & 6 & 0 & -2.91 & High & Yes & Yes & 3.13 & No \\
\hline Doxorubicin & -9.28 & -10.59 & 943.77 & 268.79 & 11 & 18 & 6 & -8.63 & Low & Yes & No & 7.64 & No \\
\hline Std values & - & - & $130-725$ & $7-200$ & - & $2-20$ & $0-6$ & - & - & - & - & $\begin{array}{l}1 \text { very } \\
\text { easy; } \\
10 \text { very } \\
\text { difficult }\end{array}$ & $\begin{array}{l}\operatorname{Max} \\
=4\end{array}$ \\
\hline
\end{tabular}

Chemistry

The title compounds were synthesized through a two-step efficient synthetic scheme. The synthetic route for the final compounds IDI 3(A-L), along with their intermediates IDI 2(A-F), is outlined in Scheme 1. The target compounds IDI 3(AL) were then successfully prepared by treating Mannich bases with primary aromatic amines, in the presence of glacial acetic acid under refluxing condition. All the synthesized intermediate compounds were characterized by melting point, FTIR and mass spectrometry. However, complete physicochemical and spectral characterizations were performed for the title compounds. The spectral data obtained were found to be in concurrence with the proposed compounds.

\section{General procedure for synthesis of compounds, IDI 2(A-F)}

Mannich bases of isatin/5-bromoisatin were prepared as per established method [44], which involved refluxing a mixture of isatin/5-bromoisatin and secondary amine (3-5) and 37\% formalin for about 8-10 hrs. The progress of the reaction 
was monitored by TLC, using methanol: chloroform (0.5:9.5) as the solvent system. After completion of the reaction, the reaction mixture was refrigerated for $48 \mathrm{hrs}$. The solid obtained was filtered, washed with $n$-hexane, dried and recrystallized from ethanol.

\section{Characterization of the synthesized intermediate compounds, IDI 2(A-F)}

\section{1-piperazin-1-ylmethyl-1H-indole-2,3-dione (IDI 2A)}

Yellowish-orange solid, yield 77\%, mp 208-210 C; IR (cm-1): 3399.04 (N-H stretch), 1215.89 (C-N stretch), 3020.19 (C-H stretch $\left(\mathrm{sp}^{2}\right)$, aromatic), $1630.55\left(\mathrm{C}=\mathrm{C}\right.$ aromatic). $\mathrm{MS}(\mathrm{m} / \mathrm{z}): 245\left(\mathrm{M}^{+}\right)$.

1-[(diphenylamino)-methyl]-1H-indole-2,3-dione (IDI 2B)

Dark orange solid, yield 52.89\%, mp 169-171 ${ }^{\circ} \mathrm{C}$; IR ( $\mathrm{cm}^{-1}$ ): 3566.87 (N-H stretch), 1319.42 (C-N stretch), 1595.18, 1441.47(C=C aromatic), $1715.89(\mathrm{C}=0) . \mathrm{MS}(\mathrm{m} / \mathrm{z}): 328\left(\mathrm{M}^{+}\right)$.

1-morpholin-4-ylmethyl-1H-indole-2,3-dione (IDI 2C)

Bright orange solid, yield $73.80 \%$, mp 196-198 ${ }^{\circ} \mathrm{C}$; IR ( $\left.\mathrm{cm}^{-1}\right)$ : 1079.60 (C-0), 1349.68 (C-N stretch), 1608.80, 1471.37 (C=C aromatic), $1715.89(\mathrm{C}=0)$. MS (m/z): $247\left(\mathrm{M}^{+}\right)$.

5-bromo-1-piperazin-1-ylmethyl-1H-indole-2,3-dione (IDI 2D)

Brown solid, yield $76.89 \%$, mp 198-200 C; IR ( $\mathrm{cm}^{-1}$ ): 3566.87 (N-H stretch), 1329.55 (C-N stretch), 1724.48 (C=0), 3184.50 (C-H stretch $\left(\mathrm{sp}^{2}\right)$ aromatic), 1612.91, 1488.85 (C=C aromatic). MS (m/z): $323\left(\mathrm{M}^{+}\right)$.

5-bromo-1-[(diphenylamino)-methyl]-1H-indole-2,3-dione (IDI 2E)

Brown solid, yield $86.60 \%$, mp 169-171 ${ }^{\circ} \mathrm{C}$; IR ( $\left.\mathrm{cm}^{-1}\right)$ : 1290.85 (C-N stretch), 3179.22 (C-H stretch (sp ${ }^{2}$ ) aromatic), 1612.10, 1468.97 (C=C aromatic), $1707.33(\mathrm{C}=0), 660.16(\mathrm{C}-\mathrm{Br}) . \mathrm{MS}(\mathrm{m} / \mathrm{z}): 406\left(\mathrm{M}^{+}\right)$.

5-bromo-1-morpholin-4-ylmethyl-1H-indole-2,3-dione (IDI 2F)

Dark orange solid, yield 86.27\%, mp 197-198 ${ }^{\circ} \mathrm{C}$; IR (cm $\left.{ }^{-1}\right)$ : 1335.48 (C-N stretch), 1608.80, 1471.37 (C=C aromatic), 1730.47 (C=0), 1092.83 (C-0), 612.07 (C-Br). MS (m/z): $325\left(\mathrm{M}^{+}\right)$.

\section{General procedure for synthesis of compounds, IDF $3(A-L)$}

Equimolar ratio of the synthesized Mannich base, IDI 2(A-F), and primary aromatic amine $(7,8)$ in the presence of catalytic amount of glacial acetic acid was refluxed for 15-18 hrs. The reaction progress was monitored by TLC using the solvent system chloroform: methanol (9:1). The reaction mixture was then cooled to obtain a solid. The solid product was filtered and recrystallized from absolute ethanol to obtain the title compound.

\section{Characterization of the synthesized title compounds, IDF 3(A-L)}

3-(Benzothiazol-2-ylimino)-1-piperazin-1-yl-1,3-dihydro-indol-2-one (IDF 3A)

Dark brown solid, yield 81\%, mp 161-163ํ․ IR ( $\left.\mathrm{cm}^{-1}\right)$ : 3345.28 (N-H stretch), 1694.46 (C=N stretch), 1599.1, 1449.45 (C=C aromatic), 1383.11 (C-N stretch). ${ }^{1} \mathrm{H}$ NMR (DMSO- $\left.d_{6}, 300 \mathrm{MHz}, \delta \mathrm{ppm}\right): 7.312$ (6H s of benzylidenimine), $7.018(5 \mathrm{H}$, $\mathrm{s}, \mathrm{CH}$ benzylidenimine), $7.622\left(4 \mathrm{H}, \mathrm{s}, \mathrm{CH}\right.$ benzylidenimine), $2.488\left(2 \mathrm{H}, \mathrm{d}, \mathrm{CH}_{2}\right.$ piperazinyl), $1.903(4 \mathrm{H}, \mathrm{s}, \mathrm{NH}) .{ }^{13} \mathrm{C} \mathrm{NMR}$ 
(DMSO- $d_{6}, 75 \mathrm{MHz}, \delta$ ppm): 125.38 (5C, s, benzothiazole), 152.80 (3’C, benzothiazole), 165.70 (3C, C=N), 124.67 (5C, s, benzylidenimine), 120.77 (7C, s, indole 2,3-dione), 56.02 (2C, d, piperazinyl). MS (m/z): 377 [M] ${ }^{+}$. Anal. Calcd. for $\mathrm{C}_{20} \mathrm{H}_{19} \mathrm{~N}_{5} \mathrm{OS}$ : C, 63.64; $\mathrm{H}, 5.07 ; \mathrm{N}, 18.55$. Found: C, 65.42; H, 4.093; N, 17.71.

\section{1-Piperazin-1-ylmethyl-3-(pyrimidin-2-yl-imino)-1,3-dihydro-indol-2-one (IDF 3B)}

Brown sticky mass, yield 81.47\%. IR ( $\mathrm{cm}^{-1}$ ): 3332.49 (N-H stretch), 1641.13 (C=N stretch), 1462.92 (C-H bend), 1716.56 $(\mathrm{C}=0) .{ }^{1} \mathrm{H}$ NMR (DMSO- $\left.d_{6}, 300 \mathrm{MHz}, \delta \mathrm{ppm}\right): 7.300$ (6H, s, benzylidenimine), 7.054 (5H, s, benzylidenimine), 4.141 (1H of methylene), $2.488\left(2 \mathrm{H}, \mathrm{d}, \mathrm{CH}_{2}\right.$ piperazinyl), 2.12 (4H, s, NH ${ }^{13} \mathrm{C}$ NMR (DMSO- $\left.d_{6}, 75 \mathrm{MHz}, \delta \mathrm{ppm}\right): 157.3$ (4C, d, pyrimidine), 164.97 (3C, C=N), 50.81 (3C, d, piperazinyl), 124.67 (5C, benzylidenimine), 122.76 (3'C, benzylidenimine). MS (m/z): 323 $[\mathrm{M}+1]^{+}$. Anal. Calcd. for $\mathrm{C}_{17} \mathrm{H}_{18} \mathrm{~N}_{6} \mathrm{O}$ : C, 63.89; $\mathrm{H}, 6.55 ; \mathrm{N}, 24.83$. Found: C, 64.32; $\mathrm{H}, 5.93 ; \mathrm{N}, 25.87$.

3-(benzothiazol-2-ylimino)-1-[(diphenylamino)-methyl]-1,3-dihydro-indol-2-one (IDF 3C)

Brown solid, yield $74.28 \%$, mp 187-189 ${ }^{\circ} \mathrm{C}$. IR ( $\left.\mathrm{cm}^{-1}\right)$ : 3010.98 (C-H aromatic), 1644.76 (C=N stretch), 1595.18,1449.47(C=C aromatic), 1285.33 (C-N). ${ }^{1} \mathrm{H}$ NMR (DMSO- $d_{6}, 300 \mathrm{MHz}, \delta$ ppm): 8.210 (4H, s, benzothiazole), $7.317(6 \mathrm{H}, \mathrm{s}$, benzylidenimine), $7.550(5 \mathrm{H}, \mathrm{d}$, benzothiazole), 7.601 (4H, s, benzylidenimine), 4.40 (1H, s, methylene), 6.547 (4H, benzene). ${ }^{13} \mathrm{C}$ NMR (DMSO- $d_{6}, 75 \mathrm{MHz}, \delta$ ppm): 164.32 (3C, imine), 117.76 (2C, d, 1-benzene), 118.14 (4C, s, 1benene), 120.85 (7C, benzylidenimine), 125.44 (5C, s, benzothiazole), 133.83 ( 7 'C, benzothiazole), 138.16 (7’C, benzylidenimine. MS (m/z): $460[\mathrm{M}]^{+}$. Anal. Calcd. for $\mathrm{C}_{28} \mathrm{H}_{20} \mathrm{~N}_{4} \mathrm{OS}$ : C, 63.02; $\mathrm{H}, 4.38 ; \mathrm{N}, 12.17$. Found: $\mathrm{C}, 62.30 ; \mathrm{H}, 3.91 ; \mathrm{N}$, 13.00. 
Table 3

In-silico toxicity prediction data of the synthesized compounds, IDF 3(A-L).

\begin{tabular}{|c|c|c|c|c|}
\hline \multirow[t]{4}{*}{ Compound } & \multicolumn{4}{|c|}{ Toxicity Prediction } \\
\hline & \multicolumn{3}{|c|}{ Osiris Property Explorer } & \multirow{3}{*}{$\begin{array}{l}\text { LAZAR* } \\
\text { Carcinogenic } \\
\text { Potency }\end{array}$} \\
\hline & Tumorigenic & Irritant & Reproductive & \\
\hline & & & effect & \\
\hline IDF 3A & None & None & None & Non-carcinogenic \\
\hline IDF 3B & None & None & None & Non-carcinogenic \\
\hline IDF 3C & None & None & None & Non-carcinogenic \\
\hline IDF 3D & None & None & None & Non-carcinogenic \\
\hline IDF 3E & None & None & None & Non-carcinogenic \\
\hline IDF 3F & None & None & None & Non-carcinogenic \\
\hline IDF $3 G$ & None & None & None & Non-carcinogenic \\
\hline IDF 3H & None & None & None & Non-carcinogenic \\
\hline IDF 3I & None & None & None & Non-carcinogenic \\
\hline IDF 3J & None & None & None & Non-carcinogenic \\
\hline IDF 3K & None & None & None & Non-carcinogenic \\
\hline IDF 3L & None & None & None & Non-carcinogenic \\
\hline
\end{tabular}

1-[(diphenylamino)-methyl]-3-(pyrimidin-2-ylimino)-1,3-dihydro-indol-2-one (IDF 3D)

Brown solid, yield $80.54 \%, \mathrm{mp} 181-183^{\circ} \mathrm{C}$. IR $\left(\mathrm{cm}^{-1}\right)$ : 1698.68 (C=N stretch), 1725.97 (C=0), 1535.16, 1451.92 (C=C aromatic), 1349.55 (C-N stretch). ${ }^{1} \mathrm{H}$ NMR (DMSO- $d_{6}, 300 \mathrm{MHz}, \delta$ ppm): 489 (2H, d, 1-benzene), 7.04 (3H, d, 1-benzene), $7.023\left(5 \mathrm{H}, \mathrm{s}\right.$, benzylidenimine), $7.618\left(4 \mathrm{H}, \mathrm{s}\right.$, benzylidenimine), $7.380\left(5 \mathrm{H}, \mathrm{s}\right.$, pyrimidine) ${ }^{13} \mathrm{C}$ NMR (DMSO- $d_{6}, 75 \mathrm{MHz}, \delta$ ppm): 159.37 (2C, amide), 164.95 (3C, C=N), 184.43 (2C, s, pyrimidine), 129.12 (4C, d, 1-benzene), 124.65 (5C, s, benzylidenimine), 138.37 (7'C, benzylidenimine), 117.06 (2C, d, 1-benzene), 129.12 (4C, s, benzylidenimine). MS (m/z): $405[\mathrm{M}]^{+}$. Anal. Calcd. for $\mathrm{C}_{25} \mathrm{H}_{19} \mathrm{~N}_{5} \mathrm{O}$ : C, 74.06; $\mathrm{H}, 4.72 ; \mathrm{N}, 12.27$. Found: $\mathrm{C}, 72.06 ; \mathrm{H}, 4.42 ; \mathrm{N}, 11.07$.

\section{3-(benzothiazol-2-ylimino)-1-morpholin-4-ylmethyl-1,3-dihydro-indol-2-one(IDF 3E)}

Orange solid, yield $70.28 \%, \mathrm{mp} 176-178^{\circ} \mathrm{C}$. IR ( $\left.\mathrm{cm}^{-1}\right): 2998.11$ (C-H stretch), 1652.76 (C=N stretch), 1595.18, 1441.47(C=C aromatic), 1285.33 (C-N stretch). ${ }^{1} \mathrm{H}$ NMR (DMSO- $d_{6}, 300 \mathrm{MHz}, \delta \mathrm{ppm}$ ): 7.613 (4H, s, benzylidenimine), $7.008(5 \mathrm{H}, \mathrm{s}$, benzylidenimine), $4.165(1 \mathrm{H}$, methylene), $7.312(6 \mathrm{H}, \mathrm{s}$, benzylidenimine), 8.386 (4H, s, benzothiazole), 2.379 $\left(2 \mathrm{H}, \mathrm{d}\right.$, tetrahydro-1,4-oxazine) $3.672\left(3 \mathrm{H}, \mathrm{d}\right.$, tetrahydro-1,4-oxazine). ${ }^{13} \mathrm{C}$ NMR (DMSO- $\left.d_{6}, 75 \mathrm{MHz}, \delta \mathrm{ppm}\right): 164.94(3 \mathrm{C}$, $\mathrm{C}=\mathrm{N}), 158.93$ (2C, amide), 122.74 (4C, s, benzothiazole), 120.77 (7C, s, benzylidenimine), 122.74 (4C, s, benzothiazole), 125.38 (5C, s, benzothiazole), 133.56 ( $7^{\prime} \mathrm{C}$, s, benzothiazole). MS (m/z): 379 [M] ${ }^{+}$. Anal. Calcd. for $\mathrm{C}_{20} \mathrm{H}_{18} \mathrm{~N}_{4} \mathrm{O}_{2} \mathrm{~S}: \mathrm{C}, 63.47$; H, 4.79; N, 14.80. Found: C, 63.13; H, 4.44; N, 13.50 . 
Orange solid, yield 50.25\%, mp 197-199 ${ }^{\circ} \mathrm{C}$. IR ( $\mathrm{cm}^{-1}$ ): 1657.85 (C=N stretch), 1383.07 (C-N stretch), 2925.26 (C-H stretch), 1062.16 (C-0). ${ }^{1} \mathrm{H}$ NMR (DMSO- $d_{6}, 300 \mathrm{MHz}, \delta$ ppm): 8.269 (4H, d, pyrimidine), 4.123 (1H, methylene), 2.370 (2H, d, tetrahydro-1,4-oxazine), 3.621 ( $3 \mathrm{H}$, d, tetrahydro-1,4-oxazine), $7.283\left(6 \mathrm{H}, \mathrm{s}\right.$, benzylidenimine). ${ }^{13} \mathrm{C}$ NMR (DMSO- $d_{6}, 75$ MHz, $\delta$ ppm): 157.85 (4C, d, pyrimidine), 164.94 (3C, C=N), 158.96 (2C, amide), 123.27 (3'C, s, benzylidenimine), 124.23 (5C, s, benzylidenimine), 138.7 ( $7^{\prime} \mathrm{C}$, s, benzylidenimine). MS (m/z): $323[\mathrm{M}]^{+}$. Anal. Calcd. for $\mathrm{C}_{17} \mathrm{H}_{17} \mathrm{~N}_{5} \mathrm{O}_{2}: \mathrm{C}, 63.15 ; \mathrm{H}$, $5.30 ; \mathrm{N}, 21.66$. Found: C, 66.28; H, 4.73; N, 20.48.

3-(benzothiazol-2-ylimino)-5-bromo-1-piperazin-1-ylmethyl-1,3-dihydro-indol-2-one (IDF 3G)

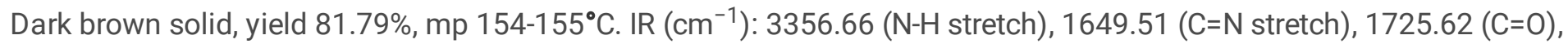
662.53 (C-Br). ${ }^{1} \mathrm{H}$ NMR (DMSO- $d_{6}, 300 \mathrm{MHz}, \delta$ ppm): 8.210 (4H, s, benzothiazole), 7.601 (7H, s, benzylidenimine), 7.478 $\left(6 \mathrm{H}, \mathrm{s}\right.$, benzylidenimine), $2.488\left(2 \mathrm{H}, \mathrm{d}\right.$, piperazine), $7.550\left(5 \mathrm{H}, \mathrm{d}\right.$, benzothiazole), $2.68(3 \mathrm{H}, \mathrm{d}$, piperazine $) .{ }^{13} \mathrm{C} N M R$ (DMSO- $d_{6}, 75$ MHz, $\delta$ ppm): 159.36 (2C, amide), 163.58 (3C, C=N), 157.99 (2C, benzothiazole), 124.68 (5C, s, benzothiazole), 117.81 (5C, benzylidenimine), 122.76 (7C, s, benzylidenimine); MS (m/z): 456 [M] ${ }^{+}$. Anal. Calcd. for $\mathrm{C}_{20} \mathrm{H}_{18} \mathrm{BrN}_{5} \mathrm{OS}$ : C, 52.64; $\mathrm{H}, 3.98 ; \mathrm{N}, 15.35$. Found: C, 52.06; $\mathrm{H}, 4.82 ; \mathrm{N}, 16.04$.

5-bromo-1-piperazin-1-ylmethyl-3-(pyrimidin-2-ylimino)-1,3-dihydro-indol-2-one (IDF 3H)

Orange solid, yield $76.34 \%, \mathrm{mp} 182-184^{\circ} \mathrm{C} . \mathrm{IR}\left(\mathrm{cm}^{-1}\right)$ : 3337.11 (N-H stretch), 1648.33 (C=N stretch), 1217.85 (C-N stretch), 652.44 (C-Br). ${ }^{1} \mathrm{H}$ NMR (DMSO-d $6,300 \mathrm{MHz}, \delta$ ppm): 7.477 (6H, s, benzylidenimine), 8.210 (4H, d, pyrimidine), 7.620 (7H, $\mathrm{s}$, benzylidenimine), 4.33 (1H, methylene), $2.494\left(2 \mathrm{H}, \mathrm{d}\right.$, piperazine), $2.77(4 \mathrm{H}, \mathrm{N}-\mathrm{H}) .{ }^{13} \mathrm{C} \mathrm{NMR}\left(\mathrm{DMSO}-d_{6}, 75 \mathrm{MHz}, \delta \mathrm{ppm}\right)$ : 119.64 (5C, s, pyrimidine), 184.47 (1C, s, pyrimidine), 164.96 (3C, C=N), 159.40 (2C, amide), 132.67 (4C, s, benzylidenimine), 117.81 (5C, s, benzylidenimine), 122.77 (7C, s, benzylidenimine), 51.26 (3C, d, piperazine). MS (m/z): 401 [M] $^{+}$. Anal. Calcd. for $\mathrm{C}_{17} \mathrm{H}_{17} \mathrm{BrN}_{6} \mathrm{O}$ : C, 50.89; H, 4.27; N, 20.94. Found: C, 52.06; H, 4.46; N, 19.05.

3-(benzothiazol-2-ylimino)-5-bromo-1-[(diphenylamino)-methyl]-1,3-dihydro-indol-2-one (IDF 3I)

Orange solid, yield $74.19 \%$, mp 156-158 ${ }^{\circ} \mathrm{C}$. IR ( $\left.\mathrm{cm}^{-1}\right)$ : 2934.96 (C-H stretch), 1645.36 (C=N stretch), 1201.49 (C-N), 648.29 (C-Br). ${ }^{1} \mathrm{H}$ NMR (DMSO- $d_{6}, 300 \mathrm{MHz}, \delta$ ppm): 8.210 (4H, s, benzylidenimine), 4.47 (1H, s, methylene), 7.431 (6H, s, benzylidenimine), 7.541 (5H, d, benzothiazole), 6.530 (4H, d, 1-benzene), 8.195 (7H, s, benzothiazole). ${ }^{13} \mathrm{C}$ NMR (DMSO$d_{6}, 75 \mathrm{MHz}, \delta$ ppm): 126.88 (6C, s, benzothiazole), 138.12 (7’C s, benzothiazole), 164.24 (3C, C=N), 158.98 (2C, amide), 125.38 (5C, benzothiazole), 117.71 (2C, d, 1-benzene), 133.77 ( $7^{\prime} \mathrm{C}, \mathrm{s}$, benzothiazole). MS (m/z): 540 [M] ${ }^{+}$. Anal. Calcd. for $\mathrm{C}_{28} \mathrm{H}_{19} \mathrm{BrN}_{4} \mathrm{OS}$ : C, 62.34; $\mathrm{H}, 3.55 ; \mathrm{N}, 10.39$. Found: $\mathrm{C}, 61.46 ; \mathrm{H}, 3.24 ; \mathrm{N}, 11.71$.

5-bromo-1-[(diphenylamino)-methyl]-3-(pyrimidin-2ylimino) 1,3-dihydro-indol-2-one (IDF 3J)

Brown solid, yield 55.60\%, mp 167-169 ${ }^{\circ} \mathrm{C}$. IR ( $\mathrm{cm}^{-1}$ ): 2998.25 (C-H stretch), 1642.27 (C=N stretch), 1201.56 (C-N stretch), 646.84 (C-Br). ${ }^{1} \mathrm{H}$ NMR (DMSO- $d_{6}, 300 \mathrm{MHz}, \delta$ ppm): 7.437 (6H, s, benzylidenimine), 7.445 (5H, s, pyrimidine), 6.554 (4H, d, 1-benzene), 4.348 (1H, s, methylene), 7.651 (7C, s, benzylidenimine). ${ }^{13} \mathrm{C}$ NMR (DMSO- $d_{6}, 75 \mathrm{MHz}, \delta$ ppm): 157.99 (4C, d, pyrimidine), 119.73 (5C, s, pyrimidine), 183.20 (1C, s, pyrimidine), 164.25 (3C, C=N), 158.98 (2C, amide), 133.78 (6C, benzylidenimine), 119.57 (4C, 1-benzene). MS (m/z): $484[\mathrm{M}]^{+}$. Anal. Calcd. for $\mathrm{C}_{25} \mathrm{H}_{18} \mathrm{BrN}_{5} \mathrm{O}: \mathrm{C}, 61.99 ; \mathrm{H}, 3.75 ; \mathrm{N}, 14.46$. Found: $\mathrm{C}, 62.76 ; \mathrm{H}, 4.02 ; \mathrm{N}, 13.77$.

3-(benzothiazol-2-ylimino)-5-bromo-1-morpholin-4-ylmethyl-1,3-dihydro-indol-2-one (IDF 3K)

Page 10/16 
Brown sticky mass, yield 81.79\%. IR (cm-1): 1386.22 (C-N stretch), 1114.31 (C-O stretch), 2937.03 (C-H stretch), 1654.34 (C=N stretch). ${ }^{1} \mathrm{H}$ NMR (DMSO- $\left.d_{6}, 300 \mathrm{MHz}, \delta \mathrm{ppm}\right): 7.551(5 \mathrm{H}, \mathrm{d}$, benzothiazole), 7.430 (6H, benzylidenimine), 7.675 (7H, benzylidenimine), $3.667(3 \mathrm{H}, \mathrm{d}$, tetrahydro-1,4-oxazine), 2.40 (2H, d, tetrahydro-1,4-oxazine), 4.032 (1H, s, methylene). ${ }^{13} \mathrm{C}$ NMR (DMSO- $d_{6}, 75 \mathrm{MHz}, \delta$ ppm): 125.47 (5C, s, benzothiazole), 133.77 (7'C, s, benzothiazole), $164.24(3 \mathrm{C}, \mathrm{C}=\mathrm{N})$, 125.36 (3'C, s, benzylidenimine), 118.77 (5C, s, benzylidenimine), 56.03 (2C, d, tetrahydro-1,4-oxazine). MS (m/z): 456 $[\mathrm{M}]^{+}$. Anal. Calcd. for $\mathrm{C}_{20} \mathrm{H}_{17} \mathrm{BrN}_{4} \mathrm{O}_{2} \mathrm{~S}$ : C, 52.52; $\mathrm{H}, 3.75 ; \mathrm{N}, 12.25$. Found: C, 52.16; $\mathrm{H}, 5.07 ; \mathrm{N}, 12.97$.

5-bromo-1-morpholin-4-ylmethyl-3-(pyrimidin-2-ylimino)-1,3-dihydro-indol-2-one (IDF 3L)

Brown sticky mass, yield 76.47\%. IR (cm $\left.{ }^{-1}\right)$ : 1303.52 (C-N stretch), 1114.29 (C-O stretch), 2937.75 (C-H stretch), 1645.08 (C=N stretch). ${ }^{1} \mathrm{H}$ NMR (DMSO- $\left.d_{6}, 300 \mathrm{MHz}, \delta \mathrm{ppm}\right): 7.426(6 \mathrm{H}, \mathrm{s}$, benzylidenimine), 7.419 (5H, pyrimidine), 7.608 (7H, s, benzylidenimine), 2.369 (2H, d, tetrahydro-1,4-oxazine), $4.126(1 \mathrm{H}, \mathrm{s}$, methylene), 3.668 (3H, d, tetrahydro-1,4-oxazine).

${ }^{13} \mathrm{C}$ NMR (DMSO- $d_{6}, 75 \mathrm{MHz}, \delta$ ppm): 157.82 (4C, d, pyrimidine), 119.73 (5C, s, pyrimidine), 164.26 (3C, C=N), 132.28 (4C, $\mathrm{s}$, benzylidenimine), 56.04 (2C, tetrahydro-1,4-oxazine), 118.81 (5C, s, benzylidenimine). MS (m/z): 401 [M] ${ }^{+}$. Anal. Calcd. for $\mathrm{C}_{17} \mathrm{H}_{16} \mathrm{BrN}_{5} \mathrm{O}_{2}$ : C, 50.76; $\mathrm{H}, 4.01 ; \mathrm{N}, 17.41$. Found: $\mathrm{C}, 49.96 ; \mathrm{H}, 4.52 ; \mathrm{N}, 16.87$.

\section{Biological Activity: Determination of percentage cell viability of MCF-7 cells by MTT assay}

The MCF-7 breast cancer cell lines were used as the cells of choice for this study. The MCF-7 cell lines were procured from NCCS, Pune. The received vials were trypsinised and transferred to a new flask. The cells were maintained in Dulbecco's modified Eagle's medium (DMEM, Hi-Media) supplemented with 10\% foetal bovine serum (FBS, Hi-Media) and $1 \%$ penicillin/streptomycin antibiotics (Hi-Media) at $37^{\circ} \mathrm{C}$, along with supply of $5 \% \mathrm{CO}_{2}$ and appropriate levels of humidity. The cells were passaged at least thrice before performing the experiment in order to get maximum viability of the cells. The cells were then seeded at a density of $1 \times 10^{5}$ cells per well on the flat bottom surface of 96 well polystyrene culture plate (Tarsons) containing $200 \mu \mathrm{L}$ of the media.

The wells were regularly replaced with fresh media and cells adhered on the tissue culture plates were observed under a bright field microscope. Cells cultured in the wells without any treatment were taken as a control. All the synthesized compounds and the standard drug, Doxorubicin, were loaded at variable concentration of $1 \mu \mathrm{g} / \mathrm{mL}, 2 \mu \mathrm{g} / \mathrm{mL}, 5 \mu \mathrm{g} / \mathrm{mL}$ and $10 \mu \mathrm{g} / \mathrm{mL}$. After incubation, the culture medium was removed from each well and a total of $100 \mu \mathrm{L}$ solution of medium and MTT ( $5 \mathrm{mg} / \mathrm{mL}$ in PBS) solution was added to each well. Incubation for $4 \mathrm{~h}$ with MTT resulted in the formation of Formazan crystals inside the wells. These were solubilized using a $100 \mu \mathrm{L}$ dimethyl sulfoxide (DMSO, Hi-Media) solution and then the optical absorbance was measured at $570 \mathrm{~nm}$ on a multimode reader (Synergy H1 hybrid, Biotek, USA). The experiment was performed in triplicate.

\section{Pharmacokinetic properties: In-silico predictions}

As poor ADME (absorption, distribution, metabolism, and excretion) profile results in failure of most of the drug candidates in clinical trials, it may result in significant surging of the drug development cost. Detection of the pharmacokinetic profile of drug candidates at an early stage thus prove significantly useful in terms of both time and resources. The SwissADME [40] and admetSAR [41, 42] programs were used to predict ADME parameters, pharmacokinetic properties and drug likeness of the synthesized compounds.

\section{Toxicity: In-silico predictions}

A number of freely available web-based programs are available to determine in-silico the toxicity of the compounds. Two such programs, OSIRIS property explorer and LAZAR were used in this study. Virtual Computational Chemistry

Page $11 / 16$ 
Laboratory maintains OSIRIS property explorer which is part of Actelion's in-house substance registration system. The software predicts various ADME properties of compounds including, toxicity parameters and drug likeness. LAZAR is a computational tool that predicts toxicological aspects such as carcinogenicity, long-term toxicity, irritability, and reproductive toxicity.

\section{Declarations}

\section{Acknowledgement}

The authors express their sincere gratitude to Central Drugs Research Institute (CDRI), Lucknow, India, for providing the library and sophisticated analytical instrument facilities. The authors are also thankful to the Central Salt and Marine Chemicals Research Institute, Bhavnagar, India for their help in elemental characterization of the compounds. The authors also acknowledge the Department of Biotechnology, IIT-BHU, Varanasi for their support in carrying out antibreast cancer activity.

\section{Conflict of Interest}

The authors declare no conflict of interest.

\section{References}

1. Yani H, Congshan S, Yun HWJ. Isatin-azole hybrids and their anticancer activities. Arch Pharm. 2019;353(1):1-10. doi: 10.1002/ardp.201900272.

2. Elzahabi HSA. Anticancer evaluation and molecular modeling of multi-targeted kinase inhibitors based pyrido[2,3d]pyrimidine scaffold. J Enzyme Inhib Med Chem. 2018;33(1):546-57. doi: 10.1080/14756366.2018.1437729.

3. Sahayarayan JJ, Rajan KS, Vidhyavathi R, Nachiappan M, Prabhu D, Alfarraj S, et al. In-silico protein-ligand docking studies against the estrogen protein of breast cancer using pharmacophore based virtual screening approaches. Saudi J Biol. Sci. 2021;28(1):400-7. doi: 10.1016/j.sjbs.2020.10.023.

4. Ozkutuk M, Ogretir C, Arslan T, Kandemirli F, Koksoy B. Acid Dissociation Constants of Some Novel Isatin Thiosemicarbazone Derivatives. J Chem Eng Data. 2010;55:2714-8. doi: 10.1021/je9009738.

5. Jarrahpour A. Synthesis, Antibacterial, Antifungal and Antiviral Activity Evaluation of Some New bis-Schiff Bases of Isatin and Their Derivatives. Molecules. 2007;12(8):1720-30. doi: 10.3390/12081720.

6. Shaikh MH, Subhedar DD, Khan FAK, Sangshetti JN, Nawale L, Arkile M, et al. Synthesis of Novel Triazoleincorporated Isatin Derivatives as Antifungal, Antitubercular, and Antioxidant Agents and Molecular Docking Study. J Het Chem. 2017;54(1):413-21. doi: 10.1002/jhet.2598.

7. Kumar S, Saha ST, Gu L, Palma G, Perumal S, Pillay AS, et al. 1H-1,2,3-Triazole Tethered Nitroimidazole-Isatin Conjugates: Synthesis, Docking, and Anti-Proliferative Evaluation against Breast Cancer. ACS Omega. 2018;3(9):12106-13. doi: 10.1021/acsomega.8b01513.

8. Al-Wabli RI, Almomen AA, Almutairi MS, Keeton AB, Piazza GA, Attia MI. New Isatin-Indole Conjugates: Synthesis, Characterization, and a Plausible Mechanism of Their in vitro Antiproliferative Activity. Drug Des Devel Ther. 2020;14:483-95. doi: 10.2147\%2FDDDT.S227862.

9. Rana S, Blowers EC, Tebbe C, Contreras JI, Radhakrishnan P, Kizhake S, Zhou T, et al. Isatin Derived Spirocyclic Analogues with alpha-Methylene-gamma-butyrolactone as Anticancer Agents: A Structure-Activity Relationship Study. J Med Chem. 2016;59(10):5121-7. doi: 10.1021/acs.jmedchem.6b00400.

10. Xie C, Tang L, Li F, Guan L. Structure-based design, synthesis, and anticonvulsant activityof isatin-1-Nphenylacetamide derivatives. Med Chem Res. 2014;23:2161-68. doi: 10.1007/s00044-013-0811-1. 
11. Shakir M, Bano N, Rauf MA, Owais M. Pharmacologically significant tetraaza macrocyclic metal complexes derived from isatin and 3,4-diaminobenzophenone: Synthesis, spectral studies and comparative in vitro biological assessment. J Chem Sci. 2017;129:1905-20. doi: 10.1007/s12039-017-1398-8.

12. Lahari K, Sundararajan R. Design and synthesis of novel isatin derivatives as potent analgesic, anti-inflammatory and antimicrobial agents. J Chem Sci. 2020;132:94. doi: 10.1007/s12039-020-01795-0.

13. Andreani A, Burnelli S, Granaiola M, Leoni A, Locatelli A, Morigi R, et al. New Isatin Derivatives with Antioxidant Activity. Eur J Med Chem. 2010;45(4):1374-78. doi: 10.1016/j.ejmech.2009.12.035.

14. Kakkar R. Isatin and its derivatives: A survey of recent syntheses, reactions, and applications. Med Chem Commun. 2019;10:351-68. doi: 10.1039/C8MD00585K.

15. Corbo F, Carocci A, Armenise D, De Laurentis N, Laghezza A, Loiodice F, et al. Antiproliferative Activity Evaluation of a Series of N-1,3-Benzothiazol-2-yl benzamides as Novel Apoptosis Inducers. J Chem. 2016:1-5 doi: $10.1155 / 2016 / 4267564$.

16. Kumar A, Shakyaa AK, Siddiqui HH. Synthesis and Anti-inflammatory Activity of Some Novel 2-Aminobenzothiazole Derivatives. Indian J Heterocycl Chem. 2016;25:243-49.

17. Meltzer-Mats E, Babai-Shani G, Pasternak L, Uritsky N, Getter T, Viskind O, et al. Synthesis and Mechanism of Hypoglycemic Activity of Benzothiazole Derivatives. J Med Chem. 2013;56(13):5335-50. doi: 10.1021/jm4001488.

18. Venugopala KN, Khedr MA, Pillay M, Nayak SK, Chandrashekharappa S, Aldhubiab BE, et al. Benzothiazole analogs as potential anti-TB agents: computational input and molecular dynamics. J Biomol Struct Dyn. 2018;37:1830-42. doi: 10.1080/07391102.2018.1470035.

19. Siddiqui N, Rana A, Khan SA, Bhat MA, Haque SE. Synthesis of benzothiazole semicarbazones as novel anticonvulsants-The role of hydrophobic domain. Bioorg Med Chem Lett. 2007;17:4178-82. doi: 10.1016/j.bmcl.2007.05.048.

20. Catalano A, Carocci A, Defrenza I, Muraglia M, Carrieri A, Bambeke FV, et al. 2-Aminobenzothiazole derivatives: Search for new antifungal agents. Eur J Med Chem. 2013;64;357-64. doi: 10.1016/j.ejmech.2013.03.064.

21. Delmas F, Avellaneda A, Giorgio CD, Robin M, Clercq ED, David PT, et al. Synthesis and antileishmanial activity of (1,3-benzothiazol-2-yl) amino-9-(10H)-acridinone derivatives. Eur J Med Chem. 2004;39:685-90. doi: 10.1016/j.ejmech.2004.04.006.

22. Ahmadi A, Khalili M, Hasanabadi J, Rezavi M, Niknafs BN. Synthesis and Antinociception activities of new substituted aminobenzothiazole analogs of lidocaine. Pharm Chem J. 2017;51:576-81. doi: 10.1007/s11094-0171656-6.

23. Joseph J, Janaki GB, Dharmaraja J. Metal complexes of 2-aminobenzothiazole derivatives as a versatile system tuning up their structural and biological properties. J Chem Pharm Res. 2016;8:133-52.

24. Singh N, Pandey SK, Anand N, Dwivedi R, Singh S, Sinha SK, et al. Synthesis, molecular modeling and bio-evaluation of cycloalkyl fused 2-aminopyrimidines as antitubercular and antidiabetic agents. Bioorg Med Chem Lett.

2011;21:4404-08. doi: 10.1016/j.bmcl.2011.06.040.

25. Chekkara R, Gorla VR, Susithra E, Sireesha R, Tenkayala SR, Rao MVB. Design, synthesis and anticancer evaluation of 2-Amino pyrimidine linked 7-Azainadzole derivatives Chem Data Coll. 2020;29:100513. doi:

10.1016/j.cdc.2020.100513.

26. Zuhal Kilic-Kurt, Nuri Ozmenb, Bakar-Ates F. Synthesis and anticancer activity of some pyrimidine derivatives with aryl urea moieties as apoptosis-inducing agents. Bioorg Chem. 2020;101:104028. doi:

10.1016/j.bioorg.2020.104028.

27. Romeo R, lannazzo D, Veltri L, Gabriele B, Macchi B, Frezza C, et al. Pyrimidine 2,4-Diones in the Design of New HIV RT Inhibitors. Molecules. 2019;24(9):1-14. doi: 10.3390/molecules24091718. 
28. Martin MW, Newcomb J, Nunes JJ, McGowan DC, Armistead DM, Boucher C, et al. Novel 2-Aminopyrimidine Carbamates as Potent and Orally Active Inhibitors of Lck. Synthesis SAR, and in Vivo Antiinflammatory Activity. J Med Chem. 2006;49(16):4981-91. doi: 10.1021/jm060435i.

29. Narwal S, Kumar S, Verma PK. Design, synthesis and antimicrobial evaluation of pyrimidin-2-ol/thiol/amine analogues. Chem Cent J. 2017;11:52.1-9. doi: 10.1186/s13065-017-0284-2.

30. Singh K, Kaur T. Pyrimidine based antimalarials: Design strategies and antiplasmodial effects. Med. Chem. Commun. 2016;7:749-68. doi: 10.1039/C6MD00084C.

31. Luo Y, Deng Y, Wang J, Long Z, Tu Z, Peng W, et al. Design, synthesis and bioevaluation of N-trisubstituted pyrimidine derivatives as potent aurora A kinase inhibitors. Eur J Med Chem. 2014:78:65-71. doi: 10.1016/j.ejmech.2014.03.027.

32. Eshkil F, Eshghi H, Saljooghi AS, Bakavoli M, Rahimizadeh M. Benzothiazole thiourea derivatives as anticancer agents: Design, synthesis, and biological screening. Russ J Bioorg Chem. 2017;43(5):576-82. doi: 10.1134/S10681620017050065.

33. Kumbhare RM, Dambal T, Kosurkar U, Sridhar V, Rao JV. Synthesis and cytotoxic evaluation of thiourea and N-bisbenzothiazole derivatives: a novel class of cytotoxic agents. Bioorg Med Chem Lett. 2012;22(1):453-55. doi: 10.1016/j.bmcl.2011.10.106.

34. Fayed EA, Eldin RRE, Mehany A, Bayoumi AH, Ammar YA. Isatin-Schiff's base and chalcone hybrids as chemically apoptotic inducers and EGFR inhibitors; design, synthesis, anti-proliferative activities and in silico evaluation. J Mol Struct. 2021;1234:130159. doi: 10.1016/j.molstruc.2021.130159.

35. Medvedev A, Buneeva O, Glover V. Biological targets for isatin and its analogues: Implications for therapy. Biologics. 2007;1(2):151.

36. de Paiva REF, Vieira EG, da Silva DR, Wegermann CA, Ferreira AMC. Anticancer compounds based on isatinderivatives: strategies to ameliorate selectivity and efficiency. Front Mol Biosci. 2020;7:627272.

37. Fatima GN, Paliwal SK, Saraf SK. Synthesis and Antimicrobial Activity of Some Novel 7-Chloro-4-aminoquinoline Derivatives. Russ J Gen Chem. 2021;91(2):285-93. doi: 10.1134/S1070363221020171.

38. Bal TR, Anand B, Yogeeswari P, Sriram D. Synthesis and evaluation of anti-HIV activity of isatin $\beta$-thiosemicarbazone derivatives. Bioorg Med Chem Lett. 2005;15(20):4451-55. doi: 10.1016/j.bmcl.2005.07.046.

39. Dong J, Wang NN, Yao ZJ, Zhang L, Cheng Y, Ouyang D, et al. ADMETlab: a platform for systematic ADMET evaluation based on a comprehensively collected ADMET database. J Cheminformatics. 2018;10(1):29. doi: 10.1186/s13321-018-0283-x.

40. Cheng F, Li W, Zhou Y, Shen J, Wu Z, Liu G, et al. admetSAR: A Comprehensive Source and Free Tool for Assessment of Chemical ADMET Properties. J Chem Inf Model. 2012;52(11):3099-105. doi: 10.1021/ci300367a.

41. Yang H, Lou C, Sun L, Li J, Cai Y, Wang Z, et al. admetSAR 2.0: web-service for prediction and optimization of chemical ADMET properties. Bioinformatics. 2018;35(6):1067-9. doi: 10.1093/bioinformatics/bty707.

42. Wong KC. Review of Spectrometric Identification of Organic Compounds. J Chem Educ. 2015;92(10):1602-03. doi: 10.1021/acs.jchemed.5b00571.

43. Jiang X, Orr BA, Kranz DM, Shapiro DJ. Estrogen induction of the Granzyme B inhibitor, proteinase inhibitor 9 , protects cells against apoptosis mediated by cytotoxic T lymphocytes and natural killer cells. Endocrinology. 2006;147(3):1419-26. doi: 10.1210/en.2005-0996.

44. Cava C, Castiglioni I. Integration of Molecular Docking and In Vitro Studies: A Powerful Approach for Drug Discovery in Breast Cancer. Appl Sci. 2020;10(19):6981. doi: 10.3390/app10196981.

\section{Figures}

Page $14 / 16$ 


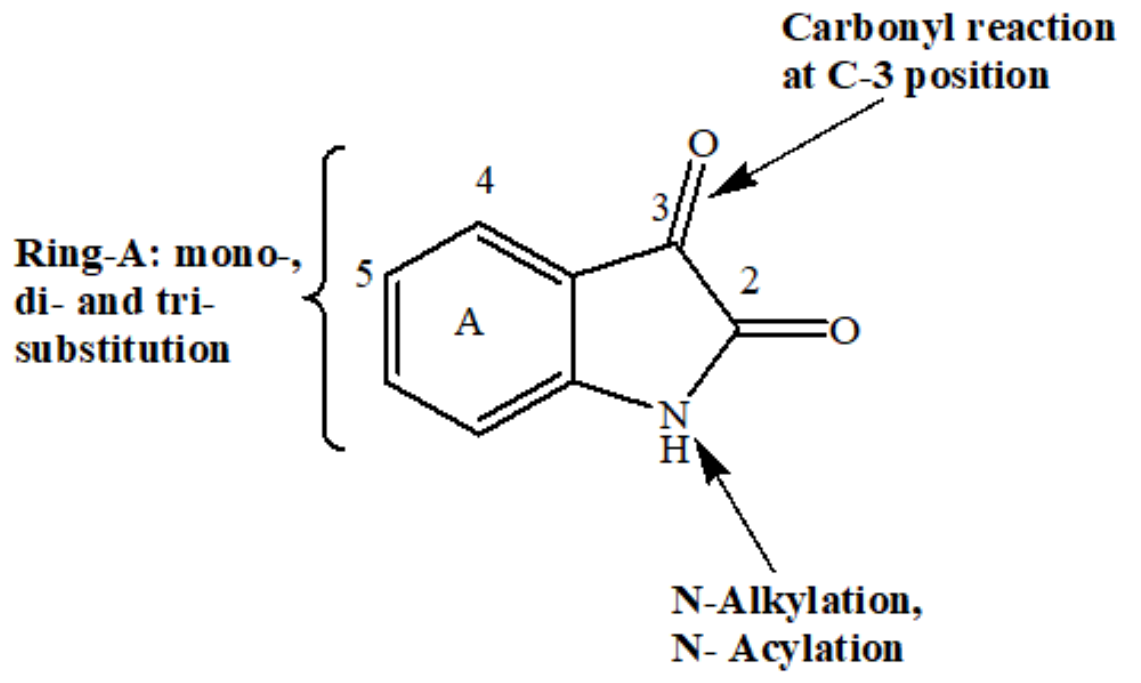

Figure 1

Isatin scaffold containing various substitution sites.
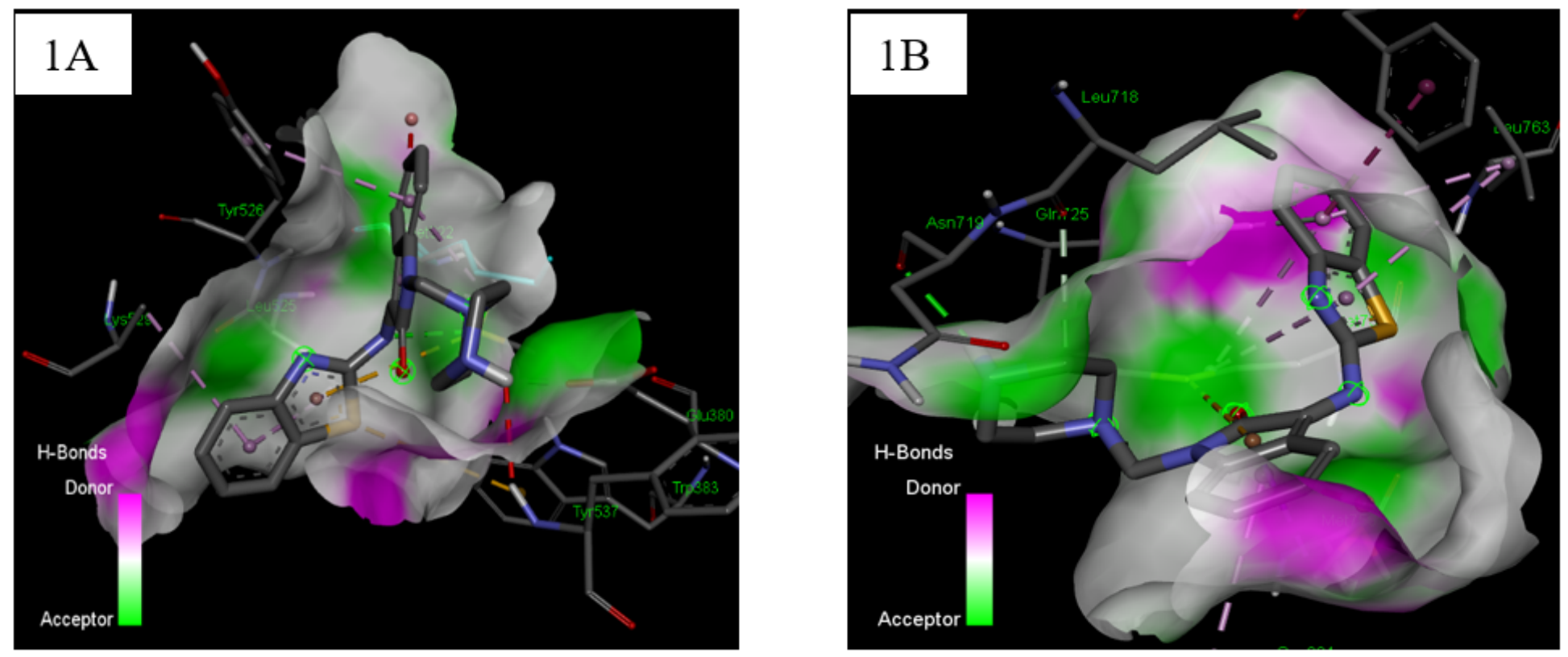

Figure 2

Depiction of ligand-receptor interaction for the potent compound IDF $\mathbf{3 G}$ in the active site of the target proteins, PDB ID: 3ERT and PDB ID: 4OAR as depicted in fig. 1A and 1B, respectively. 


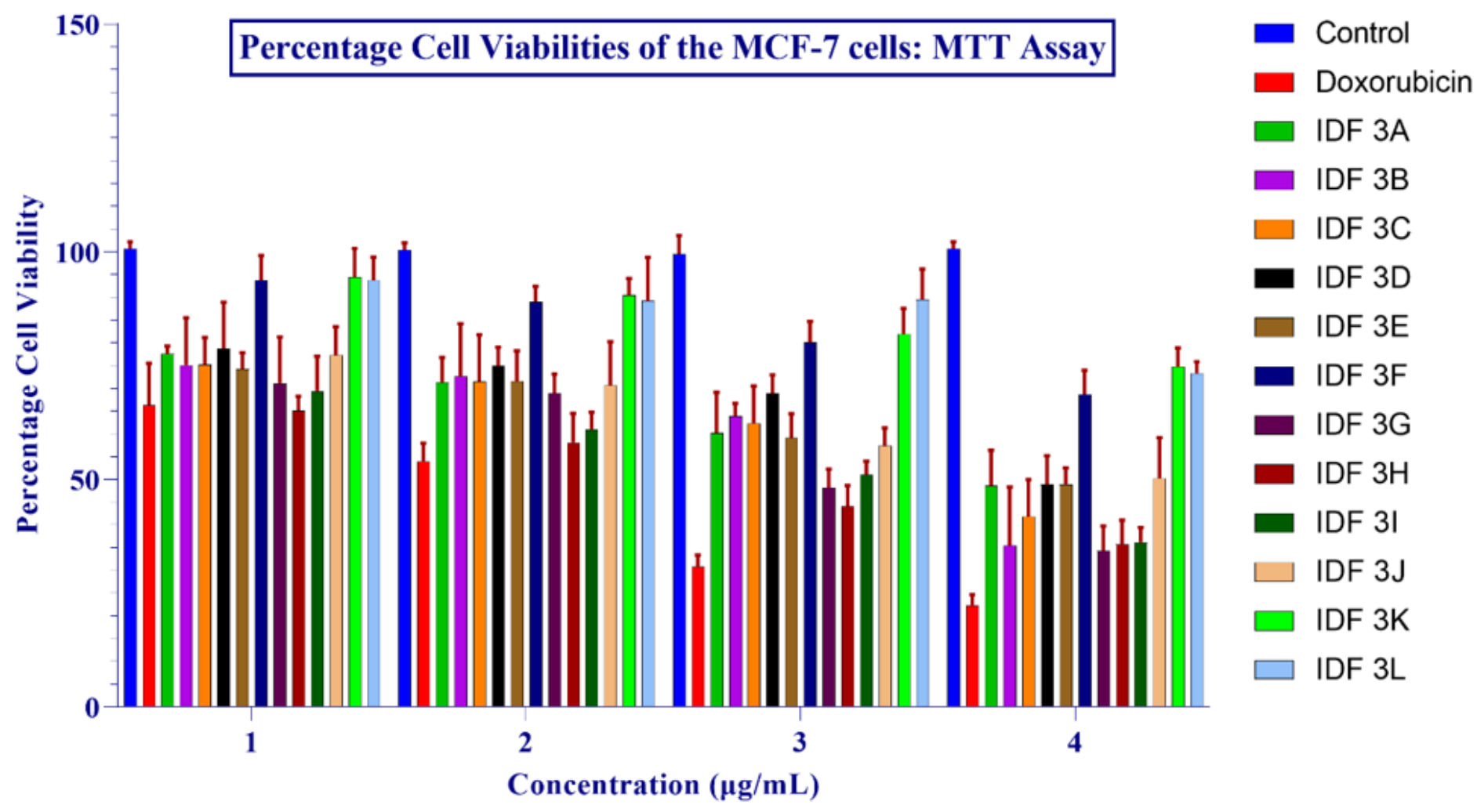

Figure 3

Graph depicting percentage cell viabilities of the MCF-7 cells on treatment with variable concentrations of the compounds, IDF $\mathbf{3}(\mathbf{A}-\mathbf{L})$ and the standard drug, Doxorubicin against the control.

\section{Supplementary Files}

This is a list of supplementary files associated with this preprint. Click to download.

- scheme1.png

- Supplementaryfile.docx 